\title{
Seismic volume attributes from time-frequency analysis
}

Hussain Syed Kazmi ${ }^{1}$, Aftab Alam², and Maryam Mahsal Khan ${ }^{3}$

\section{Abstract}

A seismic event can be characterized by three sets of attributes: (1) Wavelet attributes identify the event signal in a short time window. (2) Geometric attributes provide the event's spatial dip, azimuth, and curvatures. (3) Similarity or dissimilarity attributes measure the event's semblance or (1-semblance) with neighboring events along the geometry. Similarity and dissimilarity also are used to track horizons, faults, and channels. The resolution and fidelity of all attributes depend on spectra of both the seismic signal and noise that vary in time and space. Popular time-domain methods for attribute estimation are limited by their inability to filter frequency-dependent noise and adapt to changes in signal spectrum. The result is low resolution in narrow-band zones and poor fidelity in areas of low signal-to-noise ratio $(\mathrm{S} / \mathrm{N})$. Furthermore, time-domain tracking methods fail when signal shape or geometry changes rapidly in time and space. An alternative is to add the frequency dimension to time. It offers the flexibility to limit attribute estimation in the time-space-varying high-S/N frequency bands. The method is based on a recent patent for continuous amplitude and phase spectra (CAPS) transform, which provides high-fidelity and high-resolution estimates of the amplitude and phase spectra of a windowed signal. Furthermore, it adds an orthogonal set of uniformly sampled frequency dimensions at each time point. The orthogonal property and high-frequency resolution of Fourier coefficients allow timefrequency domain processing of all attributes. Geometry resolution can be improved by using phase difference to compute time delay, suppress noise by filtering in the frequency domain, and handle time-space variability by adapting the signal shape and geometry statistics to spectral change. Time-frequency processing improves resolution and continuity of geometric features through suppression of band-limited noise and dip-selective interfering events. Frequency-domain filtering of similarity or dissimilarity enables robust tracking of horizons and faults and mapping of channels. The sensitivity of geometry attributes that can detect geologic features in real data can be investigated. Attributes obtained using existing time-domain techniques can be compared with those derived from CAPS. The proposed attributes achieve higher resolution and better signal-to-noise ratio and continuity. Finally, applications of CAPS to robustly track horizons and faults and to map channels in three dimensions with minimum seeding and editing improve the interpreter's productivity.

\section{Introduction}

A key tool in automated interpretation is the estimate of local event coherency in a seismic data volume. Each technical advance toward automation frees interpreters from performing repetitive tasks such as horizon or fault tracking or channel mapping. Existing autotracking methods work well on data with high signal-to-noise ratio $(\mathrm{S} / \mathrm{N})$. However, they require extensive posttrack editing in areas of poor $\mathrm{S} / \mathrm{N}$.
Ever since its inception, the local similarity (coherence) attribute has been the main automation tool in the industry. Commercial applications offer variants of this attribute to speed up human interpretation. High similarity enables the tracking of continuous horizons. In contrast, low similarity, gradient discontinuity, and curvature indicate fault, channel, and fracture locations or processing artifacts and noise.

Two examples of commonly used algorithms are the coherence cube (Bahorich and Farmer, 1995) and Hilbert-transform-based instantaneous attributes (Barnes, 2007). Both use time-domain formulations of similarity to detect continuity or discontinuity of reflection events. The coherence cube uses timelagged crosscorrelation to estimate inline and crossline dips. It defines similarity as average normalized pairwise crosscorrelations of reflection events along the dip vector.

The crosscorrelation method limits the resolution of the similarity algorithm in two ways: The maximum dip is constrained by a user-defined window, and the minimum dip is constrained by the sampling interval of the data. This algorithm also suffers from poor signal-to-noise ratio and gives artificially low similarity values across zero crossings in the data in case of short window lengths. For longer window lengths, it leads to vertical striping or smeared features on section displays because of temporal averaging. A multiresolution method is required to solve this problem.

Barnes (2007) solves the problem of false dissimilarity at zero crossings by computing local dip not only from real seismic data but also by pairing with its Hilbert transform $(H t)$ in a complex analytic signal. Ht is a seismic trace that is $90^{\circ}$ phaseshifted relative to the real trace. It constitutes the imaginary part of the complex pair. Ht has no effect on the amplitude spectrum. It shifts only the phase at each frequency in the spectrum by $90^{\circ}$.

The method computes the envelope, $A(t)$, and the instantaneous phase, $\varphi(t)$, at each time sample. The instantaneous frequency, $f(t)$, is the time derivative of phase, $\varphi(t)$ It is time dependent. The use of instantaneous phase overcomes the resolution limitation inherent in correlation-based algorithms. The instantaneous-phase difference at a given frequency, $f$, between neighboring time samples of the same trace or between neighboring traces at a constant time, indexed as ${ }_{1}$ and ${ }_{2}$, provides the time delay

$$
d(t)=\frac{\varphi_{1}(t)-\varphi_{2}(t)}{2 \pi f(t)} .
$$

Although the method based on instantaneous phase solves the problem of subsample resolution, its estimate of frequency by differentiating phase becomes unstable in low-signal areas, i.e., it produces erroneous and even negative frequencies. Because the use of phase alone disregards amplitude information, erroneous estimates of instantaneous frequency caused by noise produce unreliable time delay for weak (low-amplitude) events.

This method also can be used to compute similarity. In the first step, the gradient is used to track time on neighboring
${ }^{1}$ Technical University Eindhoven.

${ }^{2}$ Prime Geoscience Corporation.
${ }^{3}$ University of Newcastle, New South Wales, Australia. http://dx.doi.org/10.1190/tle34010062.1. 
events. Then similarity is computed by plugging the $H t$ complex pair into the standard time-domain semblance formula. The pair comprises the real $(R e)$ and imaginary $(H t)$ traces in a constant $\left(t_{2}-t_{1}\right)$ window. The window moves in space by varying $t_{1}$ with $x$ following a prescribed $t-V_{\mathrm{S}}-x$ traveltime.

Semblance is the opposite of signal variance, i.e., high semblance corresponds to low variance, and vice versa. The formula for the numerator is to stack the signal first and then average the squares of stacked samples over traces. The formula for the denominator is to square the signal samples first and then stack the squares over traces. Both stacks are then summed over the number of samples in the $\left(t_{2}-t_{1}\right)$ window:

$$
S(t)=\left(\frac{1}{N}\right) * \sum_{t_{1}}^{t_{2}}\left(\frac{\left(\sum R e_{x}(t)\right)^{2}+\left(\Sigma H t_{x}(t)\right)^{2}}{\sum\left(R e_{x}(t)^{2}+H t_{x}(t)^{2}\right)}\right) .
$$

This formulation adds another degree of freedom provided by the imaginary part of the real data. A peak or trough in the real data is transformed to a zero crossing in the imaginary data, and vice versa. In practice, although this formulation solves the problem of horizontal streaking across zero crossings, it does not suppress noise, spurious edges, or erroneous attribute estimates in zones with low $\mathrm{S} / \mathrm{N}$.

\section{Proposed method}

We solve the aforementioned problems by reversing the process: We compute the real and imaginary components, frequency by frequency, in the spectral domain instead of in the time domain, as is done with instantaneous attributes. To do so, we CAPS-transform an input trace. The output comprises amplitude and phase, at a high-resolution frequency interval, at each time sample. Thus, frequency domain provides an additional tool to filter the noise, i.e., to adaptively attenuate the noise and extract attributes in high-S/N frequency bands at each sample in time and space. The use of this tool improves the quality of the desired geometric features and the confidence in their computed values.

Noise suppression to achieve higher $\mathrm{S} / \mathrm{N}$ degrades resolution. Additive random noise sets a constant base level in the amplitude spectrum. The signal spectrum rises above this level at a low-cut frequency and falls below it at a high-cut frequency. Suppression of random noise limits the bandwidth between lowand high-cut bands. This limit reduces time resolution that is directly proportional to the bandwidth. Furthermore, organized noise such as multiples produces notches in the low- to high-cut signal band. Spectral notches reduce the overall effective bandwidth of the signal and hence lower resolution.

In the following, we discuss formulations for two categories of volume attributes. The first includes geometric attributes of gradients, dip, and curvature. The second includes metrics for similarity and dissimilarity (discontinuity) among a group of neighbor events. These attributes are filtered in the frequency domain. The similarity attribute also enables the autotracking of horizons and faults with a minimum of seed points and reduces subsequent editing. Thus, it improves the interpreter's productivity.

\section{Amplitude and phase in time-frequency domain}

CAPS is a proprietary spectral-decomposition technique proposed by Alam and Taylor (2012) and by Alam (2013). It outputs amplitude and phase in two time-frequency planes for each trace in the seismic volume. It is a multiresolution transform that provides joint high resolution in time and frequency domains. Other time-frequency methods multiply the signal with a tapering window to localize it in the time domain and then compute its frequency-domain spectrum. Those methods distort the signal in the time domain and produce Gibbs oscillations and low resolution in the frequency domain.

In contrast, CAPS extrapolates short multiresolution windows into an average long window and then computes its fast Fourier transform (FFT). Thus it preserves the integrity of signal in the shortest time window and produces high-resolution frequency sampling because of the extrapolated longer time window. Key benefits of the method are high-fidelity amplitude and phase spectra and high-resolution sampling in both the time and frequency domains. There is no Gibbs oscillation because extrapolated signal automatically tapers away from the central window edges. Extrapolation uses the continuity relationship between data inside and outside the short windows. We estimate the spatial gradients using the phase information contained in these time-frequency CAPS volumes.

The combination of orthogonality, short frequency interval, $d f$, and uniform frequency sampling is unique to CAPS. For example, short-time FFT is orthogonal, but $d f=1 / d t$ is long when the time window $d t$ is short. Hence, frequency resolution is low. Wavelet-transform methods are orthogonal, but $d f$ is nonuniform and increases exponentially in octaves. The wavelet set in the dictionary of matching pursuit is generally nonorthogonal, and if the set is chosen to be orthogonal, then $d f$ is nonuniform.

\section{Gradients, dip magnitude, and azimuth}

CAPS outputs an amplitude and phase spectral pair for each frequency and for each time sample in the seismic volume. Assuming no spatial aliasing, we fix the time across several neighbor traces. The phase difference between adjacent traces at a given frequency, $f$, gives the time delay, $d t(f)$. Here, $f$ is the spectral frequency and not instantaneous frequency. The frequency-dependent gradients in the $x$ - and $y$-directions are

$$
\frac{d t(f)}{d x}=\frac{1}{2 \pi f} \frac{d \varphi(f)}{d x}, \frac{d t(f)}{d y}=\frac{1}{2 \pi f} \frac{d \varphi(f)}{d y} .
$$

Contributions to gradient calculation at frequencies with low amplitudes are filtered out by amplitude weighted averaging:

$$
\frac{d t}{d x}=\frac{1}{\sum_{f} A(f)} \sum_{f}\left(A(f) \cdot \frac{d \varphi(f)}{d x}\right), \frac{d t}{d y}=\frac{1}{\sum_{f} A(f)} \sum_{f}\left(A(f) \cdot \frac{d t(f)}{d y}\right) .
$$

The dip magnitude and azimuth follow from these directional gradients.

\section{Curvature}

Curvature attributes require first and second spatial derivatives of event times. Inputs to the second derivative are the dips along inlines and crosslines. The second derivatives of time are given by

$$
\frac{d^{2} t(f)}{d x^{2}}=\frac{d}{d x}\left(\frac{d t(f)}{d x}\right), \frac{d^{2} t(f)}{d y^{2}}=\frac{d}{d y}\left(\frac{d t(f)}{d y}\right),
$$


These derivatives are then input to compute specialized curvature attributes. Aside from large deviations in the dip at a fault edge, the gradient of the dip goes through a negative or positive extreme value, depending on whether the local surface at the edge is convex or concave upward. Thus, a curvature measure that combines the first and second derivatives can be an indicator for detecting fault or channel edge. Curvature is even more sensitive to noise than dip and produces error with the use of conventional methods. In contrast, the high-S/N band-limited spatial gradients from CAPS transform are less prone to error and allow much cleaner identification of concavity, convexity, and inflections in the data.

\section{Similarity and discontinuity}

The inline and crossline dips computed at each time sample enable tracking of event times in a neighborhood. Dip deviation called discontinuity magnitude is the mean squared difference between a planar grid fit versus actual event times over the neighborhood. Large values indicate nonplanarity and detect discontinuities.

CAPS transform provides the real, $R e$, and imaginary, $l m$, coefficients for each spectral frequency, $f$, at each time, $t$, on the fitted plane. Thus the frequency-dependent semblance at every sample point in the grid (Kazmi et al., 2012a) is

$$
S(t, f)=\left(\frac{1}{N}\right) * \sum_{t_{1}}^{t_{2}}\left(\frac{\left(\sum \operatorname{Re}_{x}(t, f)\right)^{2}+\left(\Sigma \operatorname{Im}_{x}(t, f)\right)^{2}}{\sum\left(\operatorname{Re}_{x}(t, f)^{2}+\operatorname{Im}_{x}(t, f)^{2}\right)}\right) .
$$

These frequency-dependent semblances are combined by an amplitude power-weighted measure to compute the average semblance, which then is stored at the central sample value,

$$
S(t)=\frac{\sum_{f}\left(A(t, f)^{2} * S(t, f)\right)}{\sum_{f} A(t, f)^{2}},
$$

where $A(t, f)$ is the amplitude at frequency, $f$, and sample time, $t$. This averaging step suppresses contribution to semblance from frequencies with low $\mathrm{S} / \mathrm{N}$.

\section{Discontinuity azimuth}

We postprocess the similarity volume to smooth out spurious discontinuities. Then we rotate a disk in a local sphere, centered at each sample in the processed similarity volume. The azimuth of the discontinuity is the azimuth along which this disk has the least sum. Later, we use this azimuth to predict the direction of the discontinuity. As always, the azimuth value is meaningful only when used in conjunction with the discontinuity magnitude.

\section{Frequency-tuned features}

The degree of freedom provided by the addition of frequency dimension to time enables improvement of S/N. Furthermore, it allows the extraction of frequency-tuned features that are invisible in a broadband display. The first example of
3D seismic (Figure 1) from F3 Block, Netherlands North Sea, is a comparison of the line 235 seismic section with narrow-band amplitude displays centered at 10, 25, and $42 \mathrm{~Hz}$. Details vary. Each frequency band reveals a different set of stratigraphic features (Khan, 2013). As stated earlier, although band-pass filtering enhances specific features within that band, it attenuates features in other bands and reduces overall resolution.

The second example (Figure 2), also from F3 Block, is a comparison of maximum curvature, $K_{\max }$, time slices in a CAPS broadband, 4 to $62 \mathrm{~Hz}$, and in a narrow band, 42 to $62 \mathrm{~Hz}$. The narrow band shows sharper additional fault and fracture detail (Khan, 2013).

\section{CAPS volume attributes visualized on a horizon surface}

We computed CAPS-based volume attributes on a data set from the F3 block, Netherlands North Sea. Next, we tracked a horizon, visualized it in three dimensions, and overlaid the computed attributes.

The set of displays in Figure 3 shows the sensitivity of different attributes to recognize geologic features. Figure $3 \mathrm{a}$ is a northwest-southeast $2 \mathrm{D}$ seismic section. A picked horizon shows a sequence of tilted fault blocks. Figure $3 \mathrm{~b}$ shows the picked horizon surface. Figure $3 \mathrm{c}$ shows the overlay of the amplitude attribute commonly used for locating bright spots. The combination of a structural high with bright amplitude can be a prospective candidate that requires further analysis for confirmation.

Figure $3 \mathrm{~d}$ is a dissimilarity attribute that shows faults and possible channels. Figure 3e shows dips (partial derivatives) in the crossline (northwest-southeast) direction. Partial dip in any direction aligns similar features in the orthogonal direction, so red highlights discontinuity strikes parallel to the northeastsouthwest direction. Similarly, Figure $3 f$ shows partial dips in the inline (northeast-southwest) direction and highlights discontinuity strikes trending in the northwest-southeast direction.

Figure $3 \mathrm{~g}$ combines these two dip vectors into a single dip magnitude attribute. Its color shows correct dip orientation, and lineaments amplify all faults, irrespective of strike direction. High values in dip magnitude correspond to areas where the horizon dips severely or where a fault is located. Dip can be separated from the fault by combining dip magnitude with dip deviation. A small value of

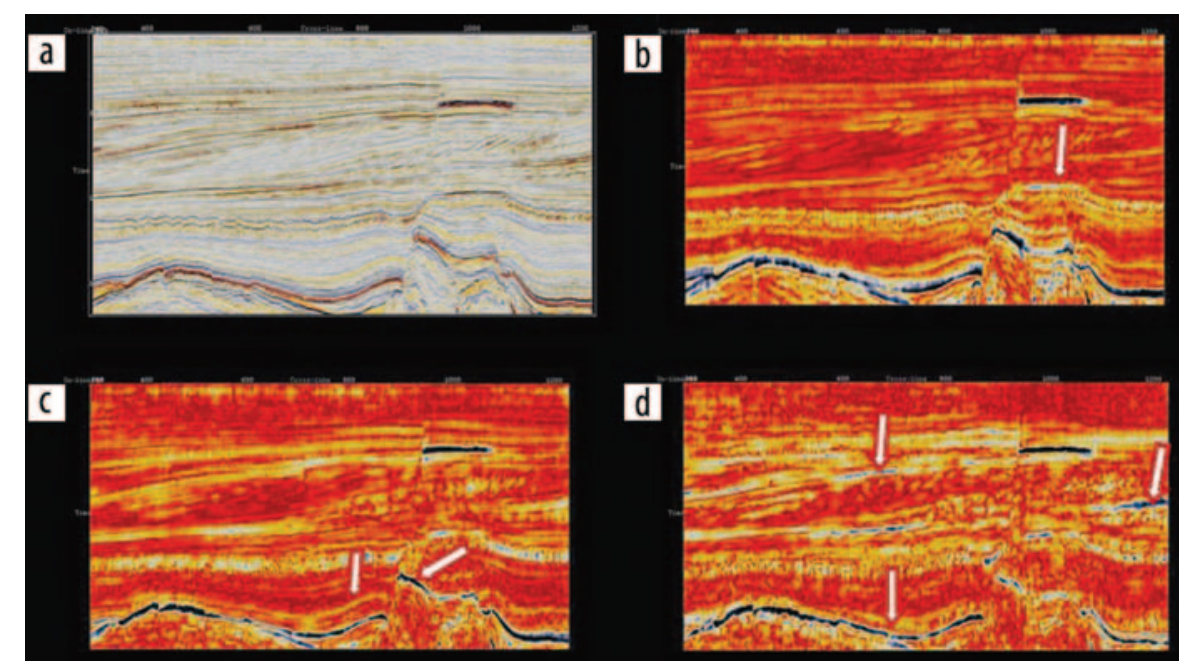

Figure 1. (a) Seismic line 235. CAPS band at (b) $10 \mathrm{~Hz}$, (c) $25 \mathrm{~Hz}$, and (d) $42 \mathrm{~Hz}$. 


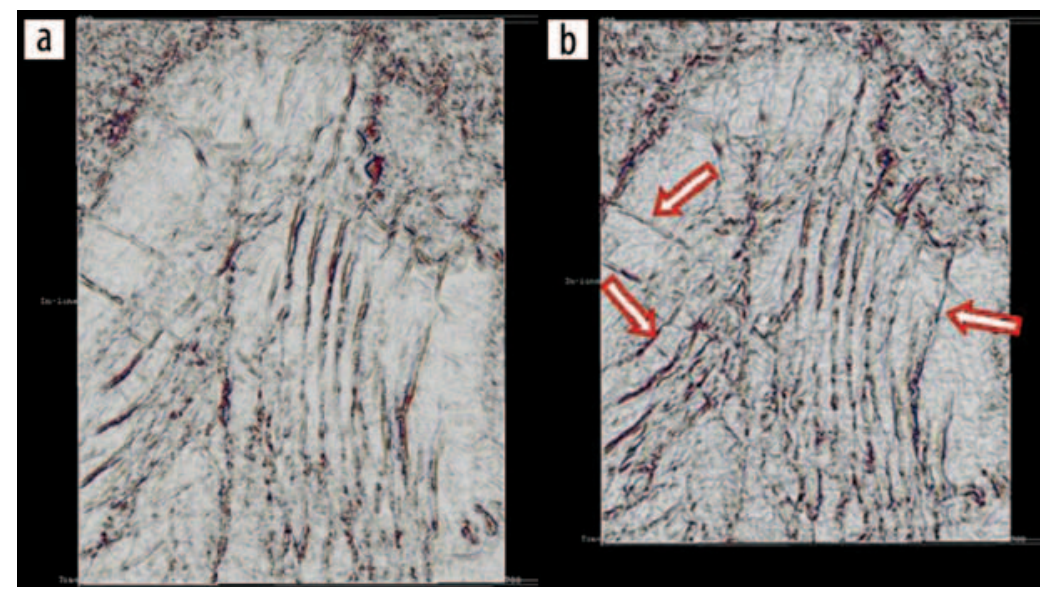

Figure 2. Maximum curvature $\left(K_{\max }\right)$ slices, CAPS band (a) 4 to $62 \mathrm{~Hz}$ and (b) 42 to $62 \mathrm{~Hz}$.
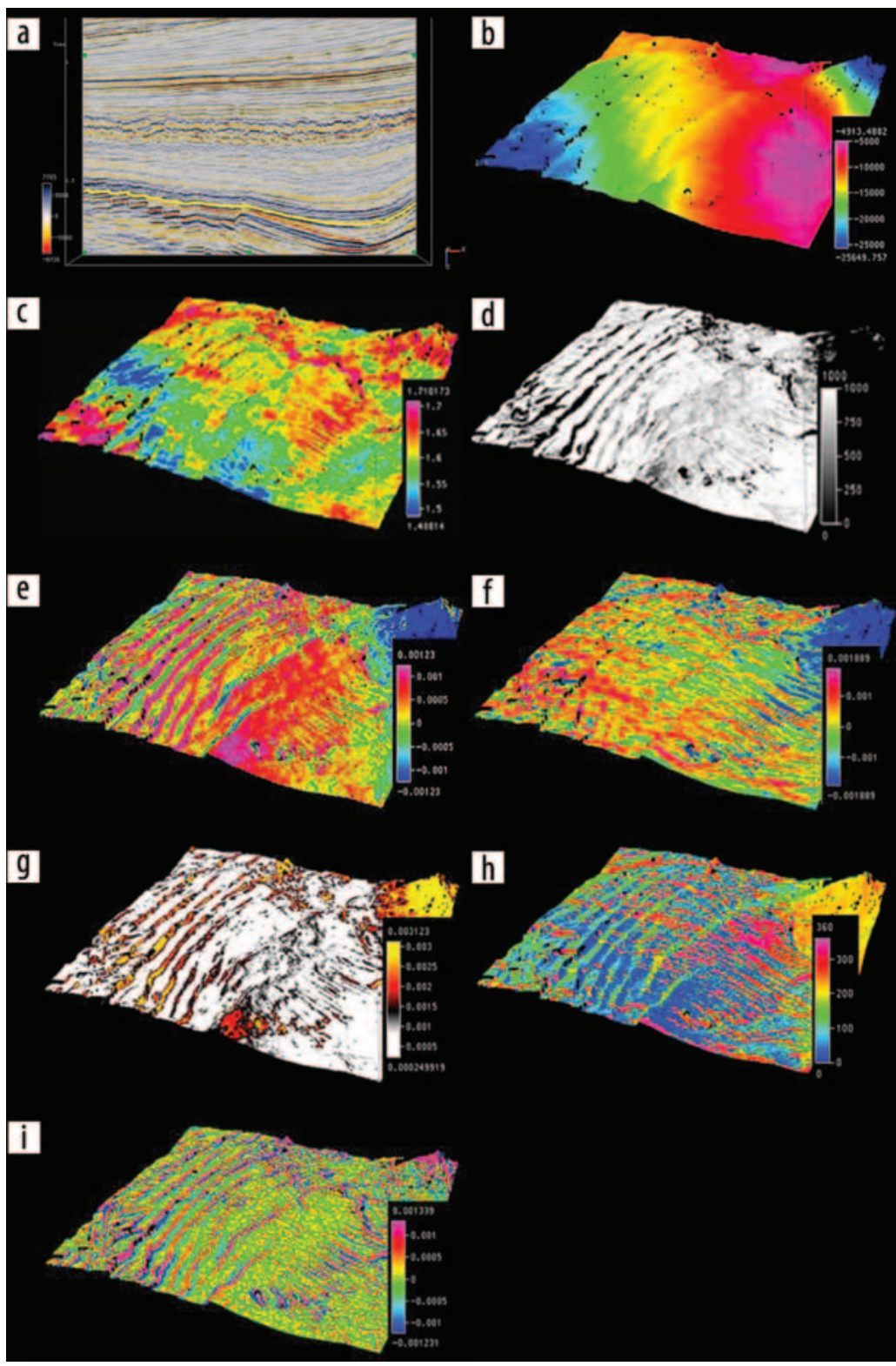

Figure 3. (a) Horizon marked on seismic section; (b) horizon time surface; (c) seismic amplitude; (d) dissimilarity; (e) dip in crossline direction; (f) dip in inline direction; (g) dip magnitude; (h) azimuth; (i) maximum curvature. dip deviation corresponds to steep planar dip, whereas large deviation corresponds to fault or high curvature.

Figure 3 h shows the discontinuity azimuth. Blue represents northeast-southwest and red represents northwest-southeast azimuths. In this example, the color patterns are parallel to crossline and inline directions and can be perceived as a combination of Figures 3e and 3f. Maximum curvature, shown in Figure 3i, not only indicates the fault trajectory in map view but also often occurs as a blue-red (magenta) pair that identifies the fault's upthrown and downthrown edges. The width of each linear pattern indicates horizontal displacement of the fault. The upthrown side, in red (or magenta), indicates convexupward curvature, and the downthrown side, in blue, indicates concave-upward curvature.

\section{Comparison with existing techniques}

Channels on time slice. We calculated the dissimilarity (1-similarity) attribute for the Stratton data set, using three algorithms. Figure 4 a shows the standard time-domain coherence-based dissimilarity. Although the channel trending northwest-southeast is discernible, the image is fuzzy and contains other features that are not so clear. Figure $4 \mathrm{~b}$ is computed using the time-domain Hilbert method. This image also is fuzzy, but intersecting events have been mostly suppressed. Figure 4c, from the CAPS time-frequency-based algorithm, shows a highly resolved laterally continuous channel without intersecting features. This improvement in detail is a result of implicit amplitude-weighted filtering of the attribute in tuned frequency bands and rejecting of noisy bands.

Fault on time slice. We computed the maximum curvature volume for the F3 data set (Kazmi et al., 2012b). Figure 5a shows the seismic time slice. Figure $5 \mathrm{~b}$ shows maximum curvature computed via the time-Hilbert algorithm introduced in previous sections. Figure $5 c$ is the equivalent image computed via CAPS time-frequency spectra followed by frequency-band-tuned signal extraction and noise suppression. Just as we observed earlier in the channel example, the image based on the timeHilbert algorithm is fuzzy and contains intersecting features. In contrast, the CAPS-based image is sharper and less noisy in the sense that it selectively clarifies features of interest.

Horizon tracking. Traditional horizon-tracking methods are error prone not only in regions of low $\mathrm{S} / \mathrm{N}$ but also when horizon dip and/or shape of the signal changes rapidly in space and where interfering signals with a different dip cross. The conventional approach to track horizons in those situations is to interactively enter multiple seed points and use a wide semblance or amplitude threshold for accepting a valid horizon. A great deal of subsequent editing 
is required to correct erroneously tracked zones and interpolate across gaps.

The use of CAPS simplifies the process. A gradient vector is computed over an initial $3-x-3$ grid. We change its size by frequencydomain processing as it adapts according to variances of amplitude, high-resolution dip, and/or signal spectrum. This greatly improves productivity because only a single seed point is needed in most situations. It also minimizes subsequent editing (Khan et al., 2012).

Figure 6a shows the initial 3-x-3 grid. We compute the local gradient, average amplitude, variance, and dominant frequency. We predict the next point, guided by the gradient and tilting the grid for maximum semblance. If the point satisfies the amplitude and dominant-frequency thresholds, we update the statistics and predict the next point. Figure $6 \mathrm{~b}$ shows the single seed point among a set of parallel sections from the Teapot Dome, Wyoming, 3D data set. Figure $6 \mathrm{c}$ is the horizon autotracked from the single seed.

Fault tracking. Generally, a fault is not confined to one horizon but cuts across many stacked events in $2 \mathrm{D}$ or $3 \mathrm{D}$ seismic. There we track the dip and/or semblance-discontinuity locations and fit a surface through them (Kazmi et al., 2012a). This feature significantly improves productivity because many traditional methods identify a fault manually on $2 \mathrm{D}$ seismic and then interpolate a surface to link the $2 \mathrm{D}$ fault segments. The angle
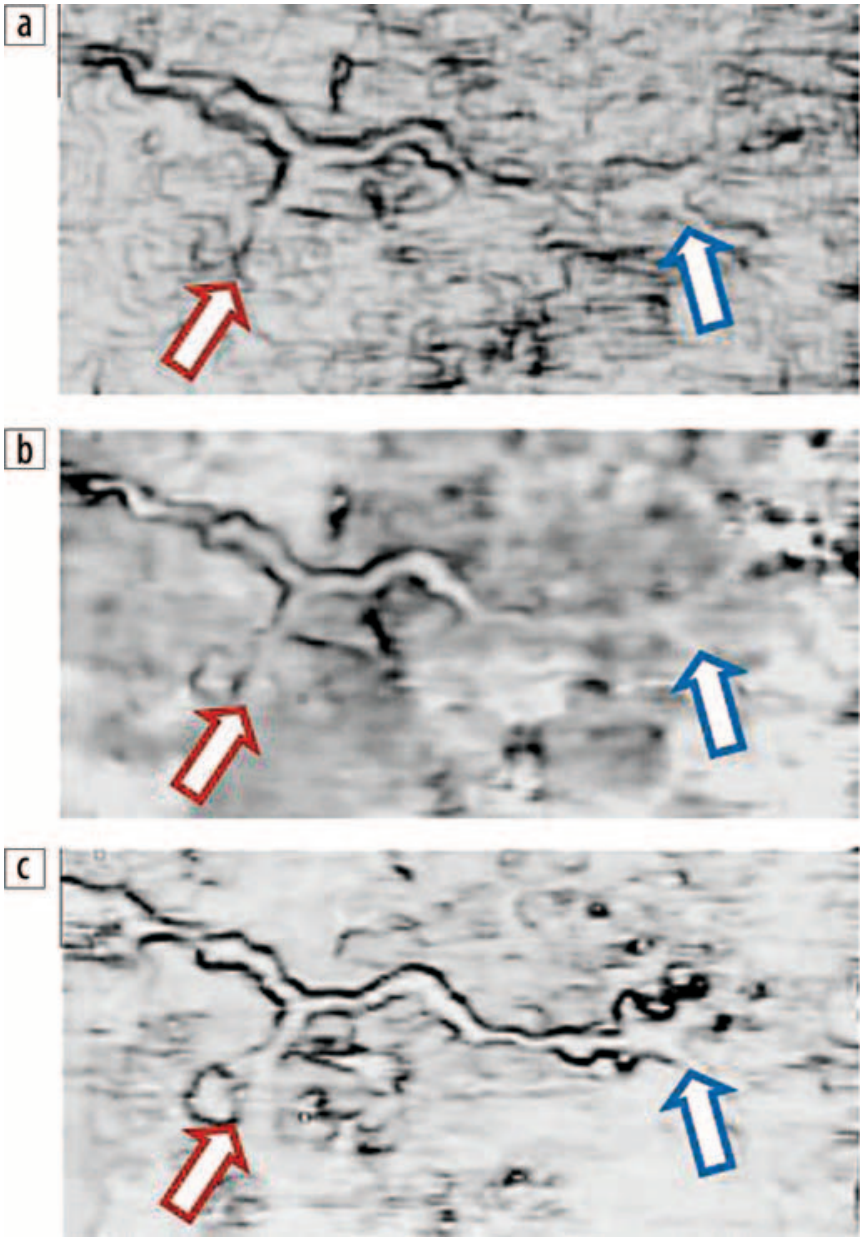

Figure 4. Dissimilarity results using (a) time domain, (b) timeHilbert domain, and (c) time-frequency domain. Red arrows mark the event delineated in image (c). Blue arrows point to the lateral continuity of the event. between the normal to the fitted surface and the vertical gives the fault angle, or hade. In addition, the curvature attributes at the fault edge provide the downward or upward direction of the fault displacement relative to the edge. Together, the fault angle, direction, and magnitude of displacement allow the estimation of the vertical and lateral displacements of the horizon resulting from the fault.

We extracted this fault semiautomatically by requiring the user to input an initial seed point. At every subsequent point, we use a gradient-descent method to traverse a path which minimizes band-limited CAPS semblance.

Figure 7a shows a seismic section and dissimilarity on a vertical section and on a time slice. Figure $7 \mathrm{~b}$ shows a fault track on a vertical section and on a time slice. Tracking was based on a single user-defined seed. Figure $7 \mathrm{c}$ shows the tracked $3 \mathrm{D}$ surface
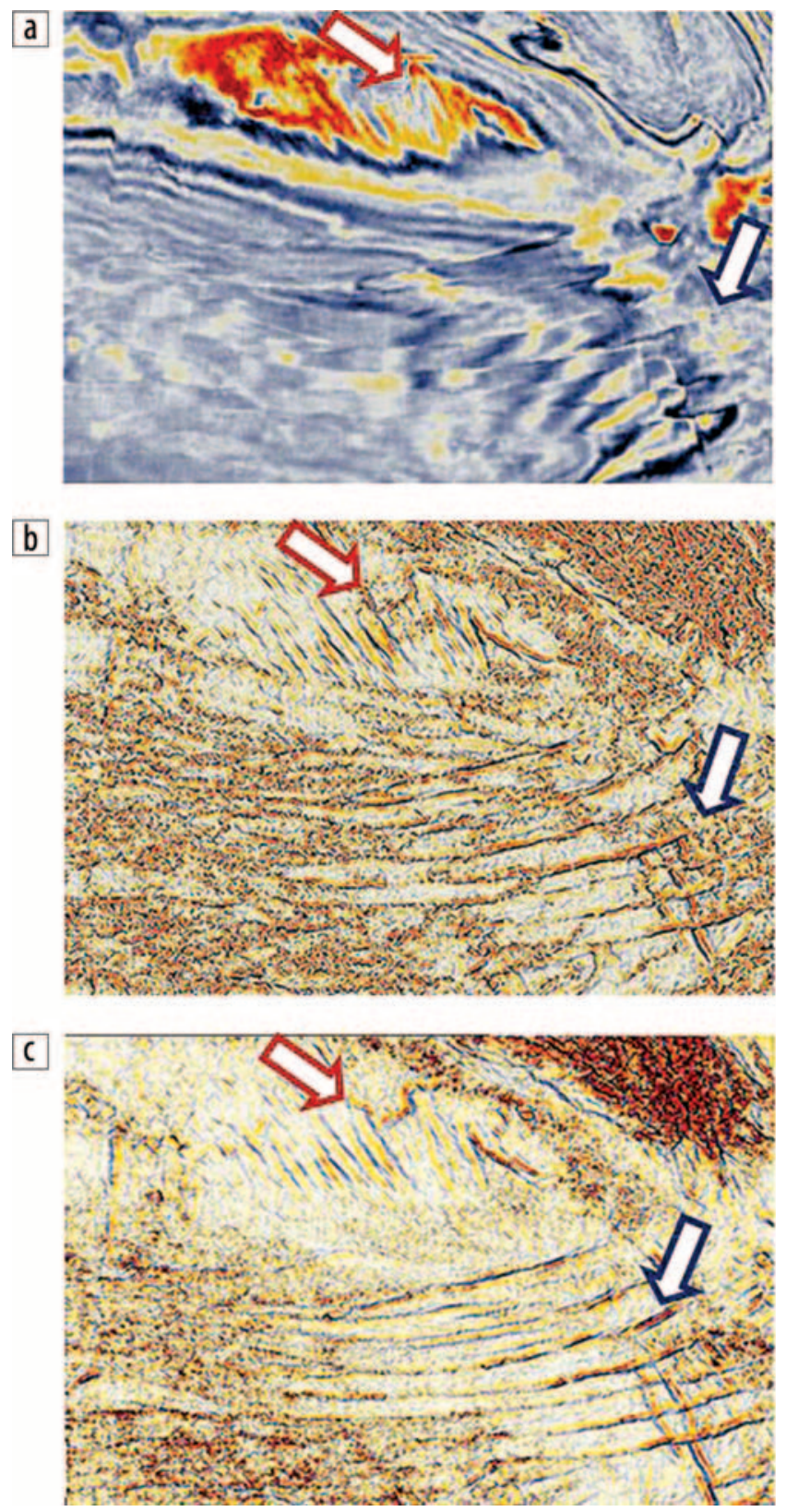

Figure 5. (a) Seismic time slice; (b) maximum curvature using time-Hilbert; (c) maximum curvature using CAPS. Red and blue arrows highlight the clarity obtained with the proposed method while suppressing noise. 
overlaid on a seismic section. The 3D fault was picked with a single seed point.

\section{Conclusion}

Continuous amplitude and phase spectra is a transform to compute an orthogonal set of Fourier coefficients with uniformly sampled high-frequency resolution from a short time-windowed

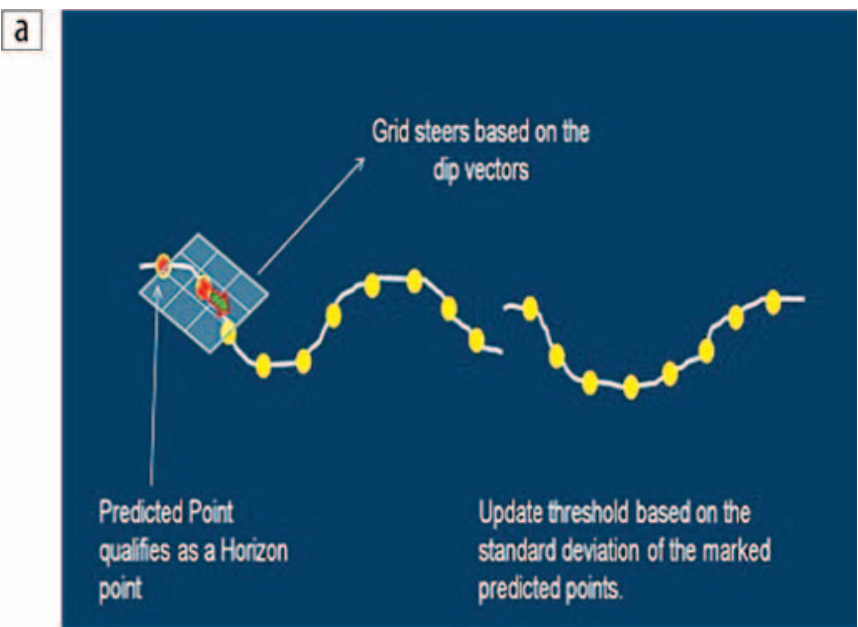

b

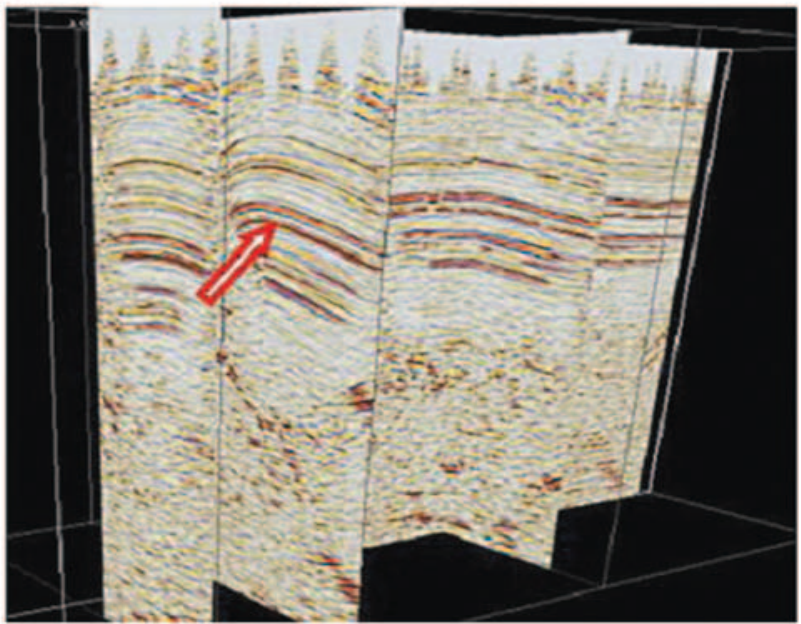

C

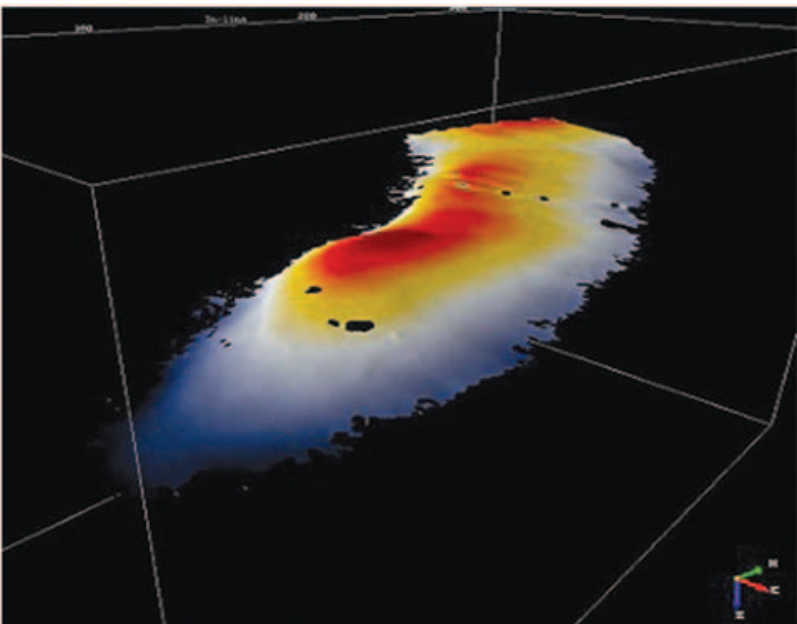

Figure 6. (a) The 3-x-3 grid estimate predicts the next point. (b) The arrow shows the seed point, Teapot Dome, Wyoming, 3D data set. (c) The entire horizon was tracked from the single seed. High amplitude is shown in red and low in blue. a

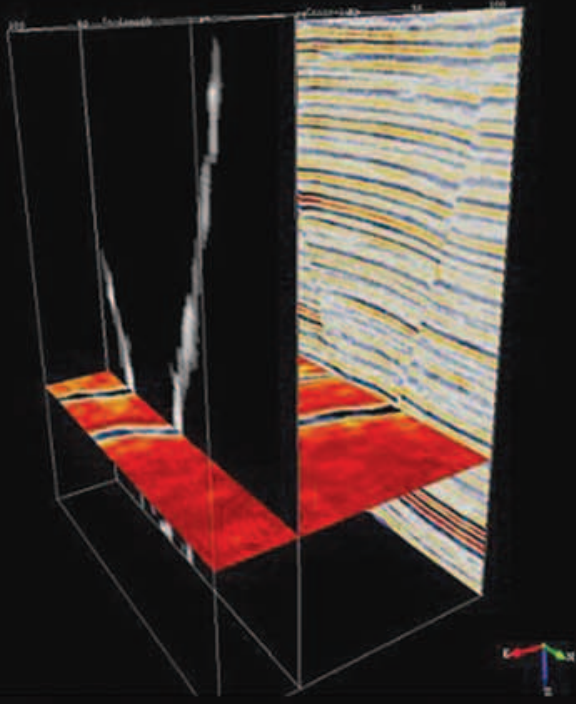

b

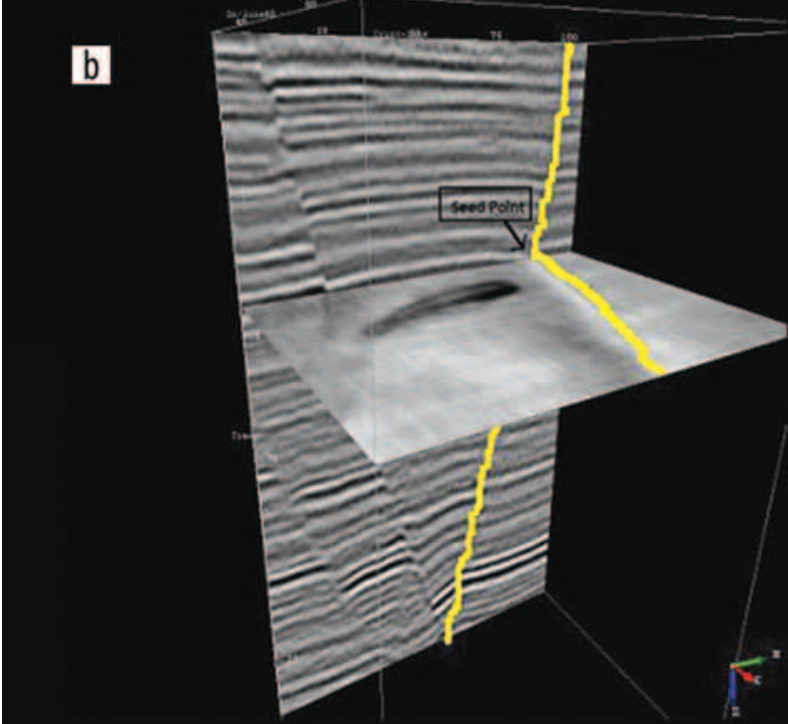

c

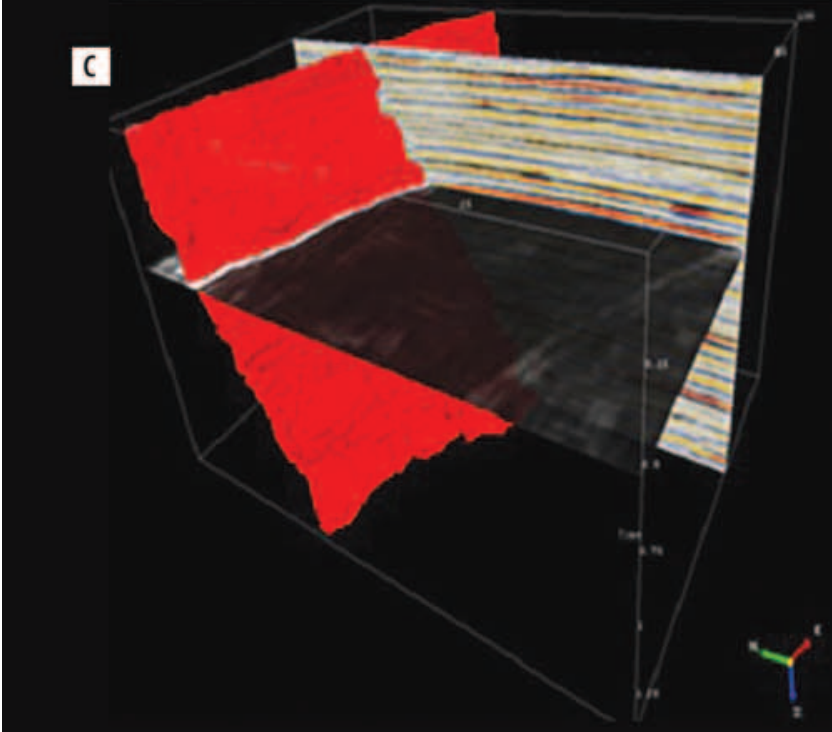

Figure 7. (a) Dissimilarity; (b) picked fault in 2D section and time slice; (c) picked fault in three dimensions. 
trace. The combination of orthogonality, uniform sampling, short frequency interval, and Gibbs oscillation-free fast Fourier transform is unique to CAPS. The higher resolution afforded by the method allows attribute extraction in tuned frequency bands and noise suppression. The result is a highly resolved and noisefree geometric feature of a selective geologic anomaly.

Comparison of images for channel and fault detection obtained with existing methods shows that images processed with CAPS time-frequency provide greater detail of a geometric feature in terms of sharpness and noise-free background than those obtained with other methods. In cases of horizon tracking and fault tracking, the proposed CAPS method improves productivity by requiring a minimum number of seed points and less subsequent editing. Frequency domain is an additional tool to selectively refine the time-domain feature in time and space. IIL

\section{Acknowledgments}

We are grateful for contributions by Sohaib Ahmad and Mumtaz Ahmad of LMKR, Islamabad, James D. Taylor of Prime Geoscience, Houston, and Tracy Stark for insightful editorial comments that helped us to improve the clarity of this article. We also gratefully acknowledge Prime Geoscience and OpenDtect for the use of their software and LMKR for the resources to perform this work. The F3 data set was provided by dGB Earth Sciences B.V., Netherlands, the Stratton data set by the Bureau of Economic Geology at the University of Texas, and the Teapot Dome 3D seismic by the U. S. Dept. of Energy, Rocky Mountain Oilfield Testing Center (RMOTC).
Corresponding author: h.s.kazmi@student.tue.nl

\section{References}

Alam, A., 2013, CAPS transform and high-resolution time-frequency spectral attributes: 83rd Annual International Meeting, SEG, Expanded Abstracts, 1374-1378, http://dx.doi. org/10.1190/segam2013-0175.1.

Alam, A., and J. D. Taylor, 2012, Time-space varying spectra for seismic processing: U. S. Patent 8,185,316.

Bahorich, M. S., and S. L. Farmer, 1995, 3-D seismic discontinuity for faults and stratigraphic features: The coherence cube: 65th Annual International Meeting, SEG, Expanded Abstracts, 93-96, http://dx.doi.org/10.1190/1.1887523.

Barnes, A. E., 2007, A tutorial on complex seismic trace analysis: Geophysics, 72, no. 6, W33-W43, http://dx.doi.org/10.1190/ 1.2785048 .

Kazmi, H. S., A. Alam, and S. Ahmad, 2012a, High resolution semblance using continuous amplitude and phase spectra: $82 \mathrm{nd}$ Annual International Meeting, SEG, Expanded Abstracts, http://dx.doi.org/10.1190/segam2012-0949.1.

Kazmi, H. S., A. Alam, S. Ahmad, and A. Shafiq, 2012b, Fault characterization using volumetric curvature from continuous phase spectrum: 82nd Annual International Meeting, SEG, Expanded Abstracts, http://dx.doi.org/10.1190/segam20120445.1.

Khan, M. M., 2013, Use of continuous amplitude phase spectrum in seismic interpretation - A case study from Netherlands and Canada: 75th Conference and Exhibition, EAGE, Extended Abstracts, http://dx.doi.org/10.3997/2214-4609.20130568. 